\title{
Super-fermion representation of quantum kinetic equations for the electron transport problem
}

\author{
Alan A. Dzhioev ${ }^{1,2}$ and D. S. Kosov ${ }^{1}$ \\ ${ }^{1}$ Department of Physics and Center for Nonlinear Phenomena and Complex Systems, \\ Université Libre de Bruxelles, Campus Plaine, CP 231, \\ Blvd du Triomphe, B-1050 Brussels, Belgium and \\ ${ }^{2}$ Bogoliubov Laboratory of Theoretical Physics, Joint Institute for Nuclear Research, RU-141980 Dubna, Russia
}

\begin{abstract}
We discuss the use of super-fermion formalism to represent and solve quantum kinetic equations for the electron transport problem. Starting with the Lindblad master equation for the molecule connected to two metal electrodes, we convert the problem of finding the nonequilibrium steady state to the many-body problem with non-Hermitian Liouvillian in super-Fock space. We transform the Liouvillian to the normal ordered form, introduce nonequilibrium quasiparticles by a set of canonical nonunitary transformations and develop general many-body theory for the electron transport through the interacting region. The approach is applied to the electron transport through a single level. We consider a minimal basis hydrogen atom attached to two metal leads in Coulomb blockade regime (out of equilibrium Anderson model) within the nonequilibrium Hartree-Fock approximation as an example of the system with electron interaction. Our approach agrees with exact results given by the Landauer theory for the considered models.
\end{abstract}

\section{INTRODUCTION}

Recently, there have been a significant progress towards theoretical and experimental understanding of electron transport through single molecules $\stackrel{1}{-\underline{4}}^{-}$The research has lead to discovery of interesting physical transport phenomena in molecules such as Franck-Condon blockade, $\underline{\underline{5}}$ nonequilibrium Kondo effect, $\underline{\underline{6}} \underline{\underline{-}}$ negative differential resistance, $\frac{9}{\underline{9}}$ vibronic effects and local heating $\underset{10}{\underline{10}}$ as well as switching and hysteresis $\underline{11}$ Being a nonequilibrium system of strongly correlated electrons molecular junstions present many interesting and challenging physical problems. Because of the very large current density (for example, if we consider a typical experimental current of a few $\mu A$ across the molecular junction of one atom wide, then the current density would be much larger than in usual mesoscopic devices) $)^{4}$ and high inhomogeneity of electron density (such as, for example, cusps on nuclear positions or electron concentrations along chemical bonds), the electron-electron correlations play a pivotal role in electron transport through molecules.

Much of the theoretical and computational studies of transport properties of molecular nanostructures are based on Landauer theory which defines the conductance from the electron transmission probability $\frac{12}{2}$ The transmission probability is usually computed by nonequilibrium Green's functions (NEGF) $\stackrel{13}{n}$ Most of the currently employed first principles electron transport calculations combine NEGF or scattering theory type approaches with ground state density functional theory (DFT) $\stackrel{14}{\underline{19}}$ There are many diverse practical implementations of NEGF-DFT and it has been applied to various molecular junctions $\stackrel{14-16,18,20-25}{-25}$ The achieving of the agreement between these first principles electron transport calculations and experiments has been elusive target for the last decade: the theoretically predicted current is systematically orders of magnitude too large $\frac{15,17,26-29}{12}$ Since reliable and accurate experimental

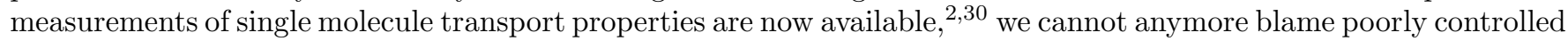
experiments for these discrepancies. All practical applications are limited to mean field, and, in spite of some recent efforts,,$\frac{31-35}{-}$ electron-electron interaction effects are difficult to include into practical calculations. Therefore, we think, it is very important at the current stage of research to move beyond currently employed NEGF-DFT scheme towards successful highly accurate methods for quantum chemical electronic structure calculations, such as, for example, coupled cluster theory $\stackrel{36,37}{ }$ and configurational interaction ${ }^{38}$ To succeed we need to develop a formalism which enables us to extend ideas of these sophisticated methods for equilibrium electronic structure calculations to nonequilibrium systems, namely, we ideally would like to reduce the problem of electron transport calculations to the eigenvalue problem with large, non-hermitian, but nevertheless finite matrix. This paper presents our first step in this direction.

The quantum kinetic equations provide a more suitable language for molecular type electronic structure calculations than NEGF or scattering theory based approaches. Recent progress in the development of kinetic equations for the electron transport presents a significant promises in this direction $\underline{\underline{39}} \underline{\underline{47}}$ Although to fully engage transport kinetic equations with the high level electronic structure calculations we need to transform them to the familiar language of second quantization, creation and annihilation operators, normal ordering, Fock space and vacuum. In this paper paper we develop a systematic scheme to convert and solve quantum kinetic equations in the language of the advanced quantum chemistry. Our derivation is based on the Lindblad master equation,,$\frac{48}{w}$ but the approach can be readily extended to other types of kinetic equations.

We would like to distance the formalism presented in this paper from the recent work of one of the authors $\stackrel{49}{\underline{4}}$ There 
was proposed a theoretical approach to the electron transport problem based on methods of equilibrium thermofield dynamics. The approach used the square root of the density matrix and employs unitary canonical transformations to build nonequilibrium Fock space. Because of that the main restriction of the method is the necessity to use the full space Liouvillian, which includes the bath continuum states, throughout the calculations. Contrary, in the current paper we develop a set of nonunitary canonical transformations which enables us to lift the above mentioned restriction.

The important remark on the notation used in the paper is the following: only creation/annihilation operators written with letter $a$ (such as for example $a_{k \alpha}$ and $a_{k \alpha}^{\dagger}$ ) are related to each other by the hermitian conjugation; all other creation $\left(c^{\dagger}, b^{\dagger}, d^{\dagger}\right.$ etc) and annihilation $(c, b, d$ etc) operators are "canonically conjugated" to each other, i.e. for example $b^{\dagger}$ does not mean $(b)^{\dagger}$ although $\left\{b, b^{\dagger}\right\}=1$.

The rest of the paper is organized as follows. In Section II, the aspects of the super-operator formalism relevant to the electron transport problem are described. We also demonstrate in this section how to solve the Lindblad master equation in the super-fermion space for a single impurity connected to one thermal bath. Section III presents the general formalism to work with the Lindblad master equation in the super-fermion Fock space for the electron transport through the interacting region. In section IV, we apply the formalism to the electron transport through single level and through molecule in Coulomb blockade regime. In this section we also compare the results with the Landauer theory. Conclusions are given in Section V. We use natural units throughout the paper: $\hbar=k_{B}=|e|=1$, where $-|e|$ is the electron charge.

\section{LIOUVILLE SPACE AND SUPEROPERATOR FORMALISM}

In this section we describe the algebraic structure of the super-fermion space relevant to the electron transport calculations. We also establish the notation and terminology which are used throughout the paper. This section is partly based in the formalism of superoperators developed by Schmutz in the context of real-time Green's functions method $^{50}$. Some aspects of the superoperator nonequilibrium Green's function theory with application to electron transport have been recently discussed by Harbola and Mukamel.$^{51}$ In contrast to Harbola and Mukamel's work, the present approach does not aim to re-formulate Keldysh NEGF in terms of super-operators and uses the formalism to solve quantum kinetic equations for the electron transport problem. We also use some ideas laid down by Prosen in his works on "third quantization" technique, $, 52,53$ where the similar method was used to solve master equation for non-interacting open Fermi systems with quadratic Hamiltonians and one dimensional spin chains.

A nonequilibrium quantum system can be described in terms of density matrix $\rho(t)$ which satisfies the Liouville equation:

$$
i \dot{\rho}(t)=[H, \rho(t)]
$$

The average value of an operator at particular moment $t$ is given by

$$
\langle A(t)\rangle=\operatorname{Tr}(\rho(t) A)
$$

For many-particle quantum systems the density matrix $\rho(t)$ and the Hamiltonian are operators in the Fock space. The Fock space can be defined by some orthonormal complete set of basis vectors:

$$
\sum_{n}|n\rangle\langle n|=I, \quad\langle n \mid m\rangle=\delta_{n m} .
$$

Let us introduce the additional Fock space which is identical copy of the initial Fock space

$$
\sum_{n}|\widetilde{n}\rangle\langle\widetilde{n}|=\widetilde{I}, \quad\langle\widetilde{n} \mid \widetilde{m}\rangle=\delta_{n m} .
$$

We denote all vectors and operators in this additional Fock space by "tilde". The vectors $|n\rangle$ and $|\widetilde{n}\rangle$ span the so-called super-Fock space, which is a direct product of the original and the "tilde" Fock spaces. Operators in this super-Fock space will be called super-operators. Let us also introduce "left vacuum vector" (why it is appropriate to call it vacuum will be clear later)

$$
|I\rangle=\sum_{n}|n\rangle \otimes|\widetilde{n}\rangle,
$$


and "nonequilibrium wavefunction":

$$
|\rho(t)\rangle=\rho(t)|I\rangle=\sum_{n m} \rho_{m n}(t)|n\rangle \otimes|\widetilde{m}\rangle,
$$

where $\rho_{m n}(t)=\langle n|\rho(t)| m\rangle$. From $\operatorname{Tr} \rho(t)=1$ it follows that the left vacuum vector and the nonequilibrium wavefunction are ortonormal to each other, $\langle I \mid \rho(t)\rangle=1$. With the use of the left vacuum and the nonequilibrium wavefunction we can re-write the average (2) as the following matrix element:

$$
\langle A(t)\rangle=\langle I|A| \rho(t)\rangle .
$$

The Liouville equation (1) becomes equivalent to the time-dependent Schrödinger equation in the super-Fock space

$$
i \frac{d}{d t}|\rho(t)\rangle=L|\rho(t)\rangle
$$

where $L=H-\widetilde{H}$ is the Liouville superoperator (Liouvillian). The left vacuum is always an eigenvector with zero eigenvalue of the Liouvillian $\langle I| L=0$, which also automatically guarantees that $\langle I \mid \rho(t)\rangle=1$.

Let us consider a system which consists of fermions distributed over $N$ levels. Let us take vector $|n\rangle$ and $|\widetilde{n}\rangle$ to be the particle number eigenstate $|n\rangle=\left|n_{1} n_{2} \ldots n_{N}\right\rangle$ and $|\widetilde{n}\rangle=\left|n_{1} \widetilde{n_{2} \ldots n_{N}}\right\rangle$

$$
\begin{aligned}
& a_{i}^{\dagger} a_{i}\left|n_{1} n_{2} \ldots n_{N}\right\rangle=n_{i}\left|n_{1} n_{2} \ldots n_{N}\right\rangle . \\
& \left.\widetilde{a} \widetilde{a}^{\dagger} \widetilde{a}_{i}\left|n_{1} \widetilde{\left.n_{2} \ldots n_{N}\right\rangle}=n_{i}\right| n_{1} \widetilde{n_{2} \ldots n_{N}}\right\rangle .
\end{aligned}
$$

Here $a_{i}^{\dagger}\left(a_{i}\right)$ are fermion creation(annihilation) operators, which satisfy the standard anticommutation relations:

$$
\left\{a_{i}, a_{j}^{\dagger}\right\}=\left\{\widetilde{a}_{i}, \widetilde{a}_{j}^{\dagger}\right\}=\delta_{i j}, \quad\left\{a_{i}, a_{j}\right\}=\left\{a_{i}^{\dagger}, a_{j}^{\dagger}\right\}=\left\{\widetilde{a}_{i}, \widetilde{a}_{j}\right\}=\left\{\widetilde{a}_{i}^{\dagger}, \widetilde{a}_{j}^{\dagger}\right\}=0,
$$

and fermionic operators in initial and tilde Fock spaces anticommute with each other. Then the left vacuum can be written as

$$
\begin{aligned}
|I\rangle & =\sum_{n_{1} n_{2} \ldots n_{N}}\left|n_{1} n_{2} \ldots n_{N}\right\rangle \otimes\left|n_{1} \widetilde{n_{2} \ldots n_{N}}\right\rangle \\
& =\exp \left(\sum_{i} a_{i}^{\dagger} \widetilde{a}_{i}^{\dagger}\right)|0\rangle \otimes|\widetilde{0}\rangle
\end{aligned}
$$

where $|0\rangle$ and $|\widetilde{0}\rangle$ are vacuums in ordinary and tilde Fock spaces (i.e. $a_{i}|0\rangle=0$ and $\widetilde{a}_{i}|\widetilde{0}\rangle=0$ for all $i$ ). The vector $|n\rangle \otimes|\widetilde{n}\rangle$ can be always multiplied by some phase factor $\exp (i \alpha)$. We found that, when one works with the fermionic systems, the convenient choice of the phase is the following

$$
\begin{aligned}
& |I\rangle=\sum_{n_{1} n_{2} \ldots n_{N}}(-i)^{n_{1}+n_{2}+\ldots+n_{N}}\left|n_{1} n_{2} \ldots n_{N}\right\rangle \otimes\left|n_{1} \widetilde{n_{2} \ldots n_{N}}\right\rangle \\
& =\exp \left(-i \sum_{i} a_{i}^{\dagger} \widetilde{a}_{i}^{\dagger}\right)|0\rangle \otimes|\widetilde{0}\rangle .
\end{aligned}
$$

One can readily demonstrate by the straightforward algebraic manipulations that

$$
a_{j}|I\rangle=-i \widetilde{a}_{j}^{\dagger}|I\rangle, \quad a_{j}^{\dagger}|I\rangle=-i \widetilde{a}_{j}|I\rangle
$$

Following the terminology of the thermofield dynamics 54,55 we will call the above relations as the "tilde conjugation rules". The above relation which transform the original operators to the tilde operators is one of the most important relations and it is used very often in our derivations in this paper. In particular, it follows from (14) that the left vacuum in the super-Fock space, $\langle I|$, is the vacuum for $a_{j}^{\dagger}-i \widetilde{a}_{j}$ and $\widetilde{a}_{j}^{\dagger}+i a_{j}$ operators. Since also $\langle I| L=0$, it is appropriate to call $\langle I|$ left vacuum vector. form

We will focus in this paper on the Lindblad quantum master equation. ${ }^{48}$ It can be written in the following general

$$
\frac{d}{d t} \rho(t)=-i[H, \rho(t)]+\sum_{\mu}\left(2 L_{\mu} \rho(t) L_{\mu}^{\dagger}-\left\{L_{\mu}^{\dagger} L_{\mu}, \rho(t)\right\}\right)
$$


where $H$ is the Hamiltonian of the system, $L_{\mu}$ is a set of generally non-Hermitian Lindblad operators that represent the influence of the environment on the system, and $\left\{L_{\mu}^{\dagger} L_{\mu}, \rho(t)\right\}$ means the anticommutator $L_{\mu}^{\dagger} L_{\mu} \rho(t)+\rho(t) L_{\mu}^{\dagger} L_{\mu}$. The Lindblad master equation is the most general master equation which can be derived under the requirements that all probabilities are real and nonnegative, $\rho(t)$ is always normalized, and $\rho(t)$ can be obtained from $\rho(0)$ by linear map $\stackrel{48,56}{ }$ The last term in the Lindblad equation is so-called dissipator. It contains the term $L_{\mu} \rho L_{\mu}^{\dagger}$ which creates quantum jumps between the states of the system and the term $\left\{L_{\mu}^{\dagger} L_{\mu}, \rho\right\}$ balances the quantum fluctuations from the quantum jumps.

Let us understand how we can re-write and solve the Lindblad master equation in super-fermion representation. We consider the following simple but nonetheless important example: A single level connected to thermal bath with temperature $T$ and chemical potential $\mu$. The Hamiltonian is

$$
H=\varepsilon a^{\dagger} a
$$

and we take Lindblad operators in the following form ${ }^{52}$

$$
L_{1}=\sqrt{\Gamma_{1}} a, \quad L_{2}=\sqrt{\Gamma_{2}} a^{\dagger} .
$$

The Lindblad equation becomes

$$
\begin{aligned}
& \frac{d}{d t} \rho(t)=-i \varepsilon\left(a^{\dagger} a \rho-\rho a^{\dagger} a\right)+\Gamma_{1}\left(2 a \rho a^{\dagger}-a^{\dagger} a \rho-\rho a^{\dagger} a\right) \\
& +\Gamma_{2}\left(2 a^{\dagger} \rho a-a a^{\dagger} \rho-\rho a a^{\dagger}\right)
\end{aligned}
$$

If we act by this equation on the left vacuum $|I\rangle$, use the tilde conjugation rules (14) and consider that the density matrix $\rho=\rho\left(a^{\dagger}, a\right)$ is the operator in original Fock space therefore it commutes with all tilde operators, we obtain the time-dependent Schrödinger equation (8) for the nonequilibrium wavefunction $|\rho(t)\rangle$ with the Liouvillian $L$ given by

$$
\begin{aligned}
L & =\varepsilon\left(a^{\dagger} a-\widetilde{a}^{\dagger} \widetilde{a}\right)-i\left(\Gamma_{1}-\Gamma_{2}\right)\left(a^{\dagger} a+\widetilde{a}^{\dagger} \widetilde{a}\right) \\
& -2\left(\Gamma_{1} \widetilde{a} a+\Gamma_{2} \widetilde{a}^{\dagger} a^{\dagger}\right)-2 i \Gamma_{2} .
\end{aligned}
$$

Within nonequilibrium thermofield dynamics a similar expression for Liouvillian (in nonequilibrium thermofield dynamics it is called Tildian) in super-Fock space was obtained axiomatically (see for example review $\underline{57}$ ).

Let us compute the average number of electrons on level $\varepsilon$ at time $t$ :

$$
n(t)=\left\langle I\left|a^{\dagger} a\right| \rho(t)\right\rangle .
$$

Differentiating this equation with respect to time we get

$$
i \frac{d}{d t} n(t)=\left\langle I\left|a^{\dagger} a L\right| \rho(t)\right\rangle .
$$

Since $\langle I| L=0$ the right side of Eq. (21) can be written as the commutator $\left\langle I\left|\left[a^{\dagger} a, L\right]\right| \rho(t)\right\rangle$. Direct calculation of this commutator with the Liouvillian (19) results into the following equation for the time-evolution of the occupation number

$$
\frac{d}{d t} n(t)=-2\left(\Gamma_{1}+\Gamma_{2}\right)\left\{n(t)-\frac{\Gamma_{2}}{\Gamma_{1}+\Gamma_{2}}\right\}
$$

which has the solution

$$
n(t)=\left(n_{0}-n_{\infty}\right) \mathrm{e}^{-2\left(\Gamma_{1}+\Gamma_{2}\right) t}+n_{\infty} .
$$

Here $n_{0}$ is the initial occupation number at $t=0, n_{\infty}=\frac{\Gamma_{2}}{\Gamma_{1}+\Gamma_{2}}$ - asymptotic occupation number at $t=\infty$. Assuming $\left|\rho_{\infty}\right\rangle$ corresponds to the equilibrium density matrix in the grand canonical ensemble, we get

$$
\left\langle I\left|a^{\dagger} a\right| \rho_{\infty}\right\rangle=f,
$$

where $f=\left[1+\exp [(\varepsilon-\mu) / T]^{-1}\right.$ is the Fermi-Dirac distribution function. So the choice of the rates $\Gamma_{1}$ and $\Gamma_{2}$ in the Lindblad operators in the form $\Gamma_{1}=\gamma(1-f), \Gamma_{2}=\gamma f$ leads to asymptotic equilibrium state in the grand canonical ensemble with temperature $T$ and chemical potential $\mu$. The parameter $\gamma=\Gamma_{1}+\Gamma_{2}$ is the relaxation rate to thermal equilibrium. The calculations along the same lines for the Lindblad master equation were also performed by Prosen within his "third quantization" method. $\underline{52}$

Let us summarize all our observation as a set of practical rules for the operations in the super-Fock space: 


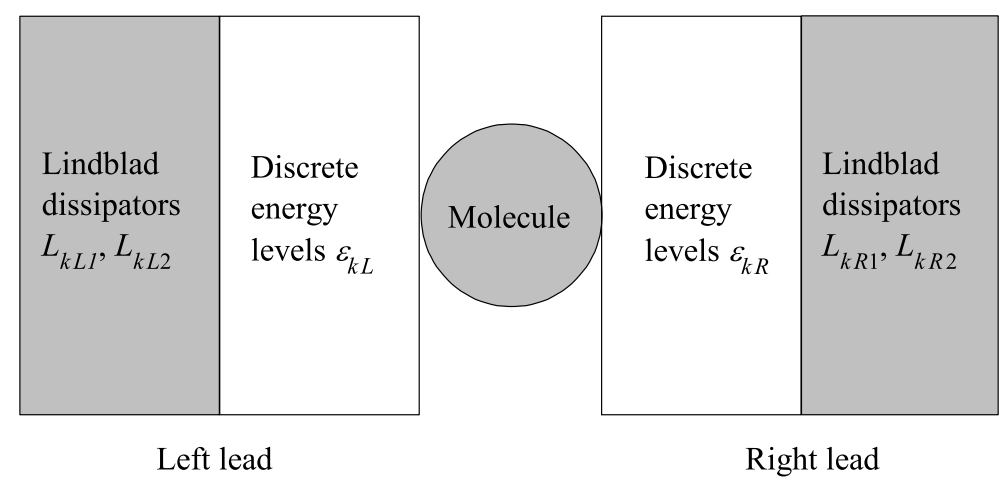

FIG. 1: Schematic illustration of the partitioning of the system. The left and right leads are assumed to be described by the non-interacting electrons, whereas electron-electron interaction is present in the molecule. Each lead is separated into two parts: The finite parts connected directly to the molecule and the macroscopically large parts (grey area) are represented by the Lindblad dissipators.

1. The left vacuum vector $|I\rangle$ and the nonequilibrium wavefunction $|\rho(t)\rangle=\rho(t)|I\rangle$ are invariant under the tilde conjugation $|\widetilde{\rho(t)}\rangle=|\rho(t)\rangle, \widetilde{|I\rangle}=|I\rangle$, and $\langle I \mid \rho(t)\rangle=1$.

2. Tilde conjugation rules: The main rule is $a_{j}|I\rangle=-i \widetilde{a}^{\dagger}|I\rangle, \quad a_{j}^{\dagger}|I\rangle=-i \widetilde{a}|I\rangle$ and as consequence the double tilde conjugation does not change the operator $\widetilde{\widetilde{A}}=A$ and $\left(c_{1} \widetilde{A+c_{2} B}\right)=c_{1}^{*} \widetilde{A}+c_{2}^{*} \widetilde{B}$.

3. Evolution of the system is described by the time-dependent Schrödinger equation $i \frac{d}{d t}|\rho(t)\rangle=L|\rho(t)\rangle$, where the Liouvillian is obtained from the corresponding master equation for the density matrix with help of the tilde conjugation rules. The nonequilibrium average is given by $\langle A(t)\rangle=\langle I|A| \rho(t)\rangle$ and $\langle I| L=0$.

\section{QUANTUM MASTER EQUATIONS FOR ELECTRON TRANSPORT THROUGH THE INTERACTING REGION IN SUPER-FERMION REPRESENTATION}

Let us now apply the formalism of superoperators discussed in the previous section to the problem of electron transport through the molecular junction. If we place a molecule into the contact with two electron reservoirs (usually metal leads) with different chemical potentials, the electric current starts to flow through it. Then, if wait for some time, which is much longer than typical electronic relaxation time of the molecule, the system will reach a nonequilibrium time-independent steady state. In this section, we will discuss some general theoretical ideas of electron transport through the molecule in nonequilibrium steady state regime based on the super-fermion representation of Lindblad master equation.

We begin with the tunneling Hamiltonian:

$$
H=H_{S}\left(a_{s}^{\dagger}, a_{s}\right)+\sum_{k, \alpha=L, R} \varepsilon_{k \alpha} a_{k \alpha}^{\dagger} a_{k \alpha}-\sum_{s, k, \alpha=L, R}\left(t_{s k \alpha} a_{k \alpha}^{\dagger} a_{s}+h . c .\right)
$$

where $a_{k \alpha}^{\dagger}\left(a_{k \alpha}\right)$ creates (destroys) an electron in the state $k$ of either the left $(\alpha=L)$ or the right $(\alpha=R)$ lead, $a_{s}^{\dagger}$ and $a_{s}$ are creation annihilation operators in the molecule, and $t_{s k \alpha}$ are the coupling parameters between the system and the leads. In what follows we assume $t_{s k \alpha}=t_{s k \alpha}^{*}$. Here $H_{S}\left(a_{s}^{\dagger}, a_{s}\right)$ represents the molecule and contains two-particle electron-electron correlations and, if necessary, electron-vibration coupling. The electron creation and annihilation operators $a_{s}^{\dagger}\left(a_{s}\right)$ and $a_{k \alpha}^{\dagger}\left(a_{k \alpha}\right)$ satisfy standard anticommutation relations. We approximate each lead by $N$ discrete single particle levels $k$ coupled to the Lindblad dissipators (see Fig. 1). We assume that if two leads were disconnected from each other, they would be in thermodynamic equilibrium with temperatures $T_{L}$ and $T_{R}$ and chemical potentials $\mu_{L}$ and $\mu_{R}$. As follows from the previous section, it can be accomplished by the use of the following set of $4 N$ Lindblad operators:

$$
L_{k \alpha 1}=\sqrt{\Gamma_{k \alpha 1}} a_{k \alpha}, \quad L_{k \alpha 2}=\sqrt{\Gamma_{k \alpha 2}} a_{k \alpha}^{\dagger}
$$

with $\Gamma_{k \alpha 1}=\gamma_{k \alpha}\left(1-f_{k \alpha}\right), \Gamma_{k \alpha 2}=\gamma_{k \alpha} f_{k \alpha}$ and $f_{k \alpha}=\left[1+\exp \left[\left(\varepsilon_{k \alpha}-\mu_{\alpha}\right) / T_{\alpha}\right]^{-1}\right.$. In the present model, the bias voltage changes only at the interface between the interacting region and the leads. We also think that a particular 
choice of the dissipators is not an issue here as long as we can place the boundary between the part of the lead which is represented by the Lindblad dissipator and part of the lead which is given by the discrete single particle levels (Fig. 1) deep enough inside the metal. This partitioning is justified in realistic systems since the screening length in the metallic leads is very short.

The Lindblad master equation is

$$
\frac{d \rho(t)}{d t}=-i[H, \rho(t)]+\sum_{k \alpha} \sum_{\mu=1,2}\left(2 L_{k \alpha \mu} \rho(t) L_{k \alpha \mu}^{\dagger}-\left\{L_{k \alpha \mu}^{\dagger} L_{k \alpha \mu}, \rho(t)\right\}\right) .
$$

In principle, this Lindblad master equation (27) for the electron transport can be obtained directly from the general full space tunneling Hamiltonian under some quite general assumptions by projecting out parts of the leads $\frac{39,41}{4}$

Now we would like to convert this Lindblad master equation to the super-Fock space. Likewise to the single-level in equilibrium considered in the previous section, we act by the Lindblad equation on the left vacuum vector $|I\rangle$, employ tilde conjugation rules and use the fact that the density matrix $\rho=\rho\left(a^{\dagger}, a\right)$ is an operator in the original Fock space therefore it commutes with all tilde operators. Then the Lindblad master equation becomes the time-dependent Schrödinger equation in the super-Fock space (8) with the following non-Hermitian Liouvillian

$$
L=L_{B}+L_{S}+L_{T}
$$

Here

$$
L_{S}=H_{S}-\widetilde{H}_{S}
$$

is the Liouvillian for the molecule and

$$
L_{T}=-\sum_{s k \alpha} t_{s k \alpha}\left(a_{k \alpha}^{\dagger} a_{s}-\widetilde{a}_{k \alpha}^{\dagger} \widetilde{a}_{s}+\text { h.c. }\right)
$$

is the Liouvillian, which describes the coupling between the molecule and the leads. They are both Hermitian. The Liouvillian for the leads

$$
L_{B}=\sum_{k \alpha} \varepsilon_{k \alpha}\left(a_{k \alpha}^{\dagger} a_{k \alpha}-\widetilde{a}_{k \alpha}^{\dagger} \widetilde{a}_{k \alpha}\right)-i \sum_{k \alpha} \Pi_{k \alpha}
$$

includes the non-Hermitian part of the Liouville operator which is responsible for the dissipation in the system:

$$
\Pi_{k \alpha}=\left(\Gamma_{k \alpha 1}-\Gamma_{k \alpha 2}\right)\left(a_{k \alpha}^{\dagger} a_{k \alpha}+\widetilde{a}_{k \alpha}^{\dagger} \widetilde{a}_{k \alpha}\right)-2 i\left(\Gamma_{k \alpha 1} \widetilde{a}_{k \alpha} a_{k \alpha}+\Gamma_{k \alpha 2} \widetilde{a}_{k \alpha}^{\dagger} a_{k \alpha}^{\dagger}\right)+2 \Gamma_{k \alpha 2} .
$$

It comprises the terms such as $a_{k \alpha} \widetilde{a}_{k \alpha}$ and $a_{k \alpha}^{\dagger} \widetilde{a}_{k \alpha}^{\dagger}$ which usually appear in the theory of superfluidity $\frac{58}{.}$ Due to these terms the structure of the nonequilibrium steady state wavefunction for the electron transport problem will have mathematical similarities with the vacuum in the Hartree-Fock-Bogoliubov theory $\underline{58}$ This terms originate from the part of the Lindblad dissipator which is responsible for the "quantum jumps" between single-particle energy levels $\varepsilon_{k \alpha}$ in the leads.

We are interested in nonequilibrium steady state situation, where the density matrix $\rho(t)$ or nonequilibrium wavefunction $|\rho(t)\rangle$ does not depend on time. Therefore, the electron transport problem is reduced to the problem of finding the eigenvector with zero eigenvalue of complex, non-Hermitian, finite-dimensional Liouville operator

$$
L\left|\rho_{\infty}\right\rangle=0 .
$$

Here $\left|\rho_{\infty}\right\rangle$ is nonequilibrium wavefunction for the nonequilibrium steady state. Subscript " $\infty$ " in $\left|\rho_{\infty}\right\rangle$ serves to emphasize that the nonequilibrium steady state can be also considered as asymptotic $(t \rightarrow \infty)$ state of the system.

Suppose we have diagonalized the Liouvillian (28) in some $\xi$-modes exactly or within some approximation

$$
L=\sum_{n}\left(\Xi_{n} \xi_{n}^{\dagger} \xi_{n}-\Xi_{n}^{*} \widetilde{\xi}_{n}^{\dagger} \widetilde{\xi}_{n}\right)
$$

Suppose also that $\langle I| \xi_{n}^{\dagger}=\langle I| \widetilde{\xi}_{n}^{\dagger}=0$. Since $\langle I| L=0$, then after diagonalization $L$ does not contain "c"-number terms. As a result a nonequilibrium steady state can be determined as a vacuum of $\xi$-modes. The creation and annihilation operators of $\xi$-modes will not be Hermitian adjoint to each other, since the left and right vacuums are different. If we recall that the basic idea of standard (equilibrium) quasiparticles is to represent true ground state of interacting many particle systems as a vacuum with respect to some quasiparticle annihilation operators $\stackrel{59,60}{\longleftarrow}$ then these $\xi$-modes can 
be regarded as nonequilibrium quasiparticles. The nonequilibrium quasiparticles include nonequilibrium, correlations and dissipation into their structure. This nonequilibrium quasiparticle description is, in principle, exact if one defines the quasiparticles in terms of the exact eigenstates of the many-particle Liouvillian. Although in practice it can be done approximately by establishing the relation between quasiparticles and "bare" particles of the system, for example, by canonical transformations. Below, we demonstrate how it can be done.

Let us begin diagonalization of the Liouvillian (28). First, we separate the part of the Liouvillian which contains only operators from the leads $L_{B}$. It is not Hermitian due to the presence of the dissipators. Direct calculations show that the following canonical (but not unitary) transformation

$$
\begin{aligned}
& a_{k \alpha}=b_{k \alpha}-i f_{k \alpha} \widetilde{b}_{k \alpha}^{\dagger}, \quad \widetilde{a}_{k \alpha}=\widetilde{b}_{k \alpha}+i f_{k \alpha} b_{k \alpha}^{\dagger}, \\
& a_{k \alpha}^{\dagger}=\left(1-f_{k \alpha}\right) b_{k \alpha}^{\dagger}+i \widetilde{b}_{k \alpha}, \quad \widetilde{a}_{k \alpha}^{\dagger}=\left(1-f_{k \alpha}\right) \widetilde{b}_{k \alpha}^{\dagger}-i b_{k \alpha},
\end{aligned}
$$

with $f_{k \alpha}=\Gamma_{k \alpha 2} /\left(\Gamma_{k \alpha 1}+\Gamma_{k \alpha 2}\right)=\left[1+\exp \left[\left(\varepsilon_{k \alpha}-\mu_{\alpha}\right) / T_{\alpha}\right]^{-1}\right.$, brings $L_{B}$ to the diagonal form

$$
L_{B}=\sum_{k \alpha}\left\{E_{k \alpha} b_{k \alpha}^{\dagger} b_{k \alpha}-E_{k \alpha}^{*} \widetilde{b}_{k \alpha}^{\dagger} \widetilde{b}_{k \alpha}\right\}
$$

Here $E_{k \alpha}=\varepsilon_{k \alpha}-i\left(\Gamma_{k \alpha 1}+\Gamma_{k \alpha 2}\right)=\varepsilon_{k \alpha}-i \gamma_{k \alpha}$ and $E_{k \alpha}^{*}=\left(E_{k \alpha}\right)^{*}$ is complex conjugated energy. Nonunitary canonical transformations mean that these transformations, although being nonunitary, preserve the anticommutation relation between the fermion creation/annihilation operators. Since, $b_{k \alpha}^{\dagger}=a_{k \alpha}^{\dagger}-i \widetilde{a}_{k \alpha}$, the vector $\langle I|$ is automatically the vacuum for $b_{k \alpha}^{\dagger}$ and $\widetilde{b}_{k \alpha}^{\dagger}$ operators. We would like to repeat the important remark on the notation we use: only creation/annihilation operators written with letter $a$ (such as, for example, $a_{k \alpha}$ and $a_{k \alpha}^{\dagger}$ ) are related to each other by Hermitian conjugation; all other creation $\left(b^{\dagger}, c^{\dagger}\right.$, etc.) and annihilation operators $(b, c$, etc.) are "canonically conjugated" to each other, i.e. for example $b^{\dagger}$ does not mean $(b)^{\dagger}$ although $\left\{b, b^{\dagger}\right\}=1$.

Having reduced the reservoir part to the ideal gas of non-Hermitian fermions, let us begin to work with Liouvillian of the interacting region. We take the Hamiltonian for the molecule in the following general form

$$
H_{S}=\sum_{s_{1} s_{2}} K_{s_{1} s_{2}} a_{s_{1}}^{\dagger} a_{s_{2}}+\frac{1}{4} \sum_{s_{1} s_{2} s_{3} s_{4}} V_{s_{1} s_{2} s_{3} s_{4}} a_{s_{1}}^{\dagger} a_{s_{2}}^{\dagger} a_{s_{4}} a_{s_{3}}
$$

where $K_{s_{1} s_{2}}$ is single-particle matrix element which contains kinetic energy, electron-nuclei attraction and interaction with external fields such as, for example, gate voltage, $V_{s_{1} s_{2} s_{3} s_{4}}$ is antisymmetrized matrix elements of Coulomb electron-electron interactions. Using the Wick theorem we perform the normal ordering of the Liouvillian for the interacting region:

$$
L_{S}=H_{S}-\widetilde{H}_{S}=L_{S}^{(0)}+L_{S}^{\prime},
$$

where $L_{S}^{(0)}$ is the quadratic part

$$
L_{S}^{(0)}=\sum_{s_{1} s_{2}}\left(K_{s_{1} s_{2}}+\sum_{s_{3} s_{4}} n_{s_{4} s_{3}} V_{s_{1} s_{3} s_{2} s_{4}}\right): a_{s_{1}}^{\dagger} a_{s_{2}}: \text {-t.c. }
$$

and $L_{S}^{\prime}$ contains part of the electron-electron interaction, which is irreducible to the quadratic form :

$$
L_{S}^{\prime}=\frac{1}{4} \sum_{s_{1} s_{2} s_{3} s_{4}}\left(V_{s_{1} s_{2} s_{3} s_{4}}: a_{s_{1}}^{\dagger} a_{s_{2}}^{\dagger} a_{s_{4}} a_{s_{3}}:- \text { t.c. }\right) \text {. }
$$

The steady state single-particle density matrix is $n_{s_{2} s_{1}}=\left\langle I\left|a_{s_{1}}^{\dagger} a_{s_{2}}\right| \rho_{\infty}^{(0)}\right\rangle$, and the notation (t.c.) means the tilde conjugation (i.e. $a_{s} \rightarrow \widetilde{a}_{s}, n_{s_{2} s_{1}} \rightarrow n_{s_{2} s_{1}}^{*}$, etc.). The normal ordering is asymmetric: it is performed with respect to the left vacuum $\langle I|$ from the left and nonequilibrium vacuum $\left|\rho_{\infty}^{(0)}\right\rangle$, which is the solution of the following eigenvalue problem

$$
L^{(0)}\left|\rho_{\infty}^{(0)}\right\rangle=0,
$$

from the right. Here

$$
L^{(0)}=L_{S}^{(0)}+L_{T}+L_{B}
$$


is the quadratic part of the Liouvillian. We would like to comment on the applicability of the Wick theorem to our case, when the left vacuum state is not the same as the right vacuum. As it will be demonstrated below, we can always write operators $a_{s}, a_{s}^{\dagger}$ as a linear combinations of some fermionic creation and annihilation operators (let us call them $c_{n}$ ) in such a way that $c_{n}\left|\rho_{\infty}^{(0)}\right\rangle=\widetilde{c}_{n}\left|\rho_{\infty}^{(0)}\right\rangle=0$, and $\langle I| c_{n}^{\dagger}=\langle I| \widetilde{c}_{n}^{\dagger}=0$. In this case, the Wick theorem is applicable $\frac{61}{\underline{1}}$ and the Liouvillian can be brought to the normal form with respect to the "left vacuum" $\langle I|$ from the left and the vacuum for the quadratic part of the Liouvillian $\left|\rho_{\infty}^{(0)}\right\rangle$ (41) from the right.

Since $L_{S}^{(0)}$ is quadratic and Hermitian, it can be diagonalized exactly by unitary transformation $\mathbf{D}$ of creation and annihilation operators

$$
a_{s}=\sum_{s^{\prime}} D_{s s^{\prime}} \alpha_{s^{\prime}}
$$

Note, that $\mathbf{D}$ does not mix nontilde and tilde operators. To diagonalize (39), $L_{S}^{(0)}=\sum_{s} \varepsilon_{s}\left(\alpha_{s}^{\dagger} \alpha_{s}-\widetilde{\alpha}_{s}^{\dagger} \widetilde{\alpha}_{s}\right)$, the matrix D should satisfy the following equation

$$
\sum_{s_{2}}\left(K_{s_{1} s_{2}}+\sum_{s_{3} s_{4}} n_{s_{4} s_{3}} V_{s_{1} s_{3} s_{2} s_{4}}\right) D_{s_{2} s}=\varepsilon_{s} D_{s_{1} s}
$$

This is the nonlinear eigenproblem, since $n_{s_{4} s_{3}}$ depends on $\mathbf{D}$ and $\varepsilon_{s}$. Next, we introduce system operators $b_{s}^{\dagger}=\alpha_{s}^{\dagger}-i \widetilde{\alpha}_{s}, \widetilde{b}_{s}^{\dagger}=\widetilde{\alpha}_{s}^{\dagger}+i \alpha_{s}$ which annihilate left vacuum $\langle I|$ due to tilde conjugation rules. The canonically conjugate annihilation operators are $b_{s}=\alpha_{s}, \widetilde{b}_{s}=\widetilde{a}_{s}$. Then the Liouvillian takes the form

$$
L=\sum_{s} \varepsilon_{s}\left(b_{s}^{\dagger} b_{s}-\widetilde{b}_{s}^{\dagger} \widetilde{b}_{s}\right)+L_{B}+L_{T}+L_{S}^{\prime}=L^{(0)}+L_{S}^{\prime},
$$

where the tunneling interaction is expressed in terms of new creation and annihilation operators $b_{k \alpha}, b_{s}$

$$
L_{T}=-\sum_{s k \alpha} T_{s k \alpha}\left\{\left(b_{k \alpha}^{\dagger} b_{s}+b_{s}^{\dagger} b_{k \alpha}\right)-\left(\widetilde{b}_{k \alpha}^{\dagger} \widetilde{b}_{s}+\widetilde{b}_{s}^{\dagger} \widetilde{b}_{k \alpha}\right)+i f_{k \alpha}\left(\widetilde{b}_{k \alpha}^{\dagger} b_{s}^{\dagger}+b_{k \alpha}^{\dagger} \widetilde{b}_{s}^{\dagger}\right)\right\}
$$

and $T_{s k \alpha}=\sum_{s^{\prime}} t_{s^{\prime} k \alpha} D_{s s^{\prime}}$ are renormalized matrix elements of the tunneling interaction. After the transformation the tunneling part of the Liouvillian depends on temperatures and chemical potentials of the leads through the Fermi-Dirac occupation numbers $f_{k \alpha}$.

Next we diagonalize the quadratic part of (45), $L^{(0)}$, exactly,

$$
L^{(0)}=\sum_{n}\left(\Omega_{n} c_{n}^{\dagger} c_{n}-\Omega_{n}^{*} \widetilde{c}_{n}^{\dagger} \widetilde{c}_{n}\right)
$$

It results to the nonequilibrium Hartree-Fock theory for the electron transport problem. Creation and annihilation operators $c_{n}^{\dagger}, c_{n}$ can be regarded as nonequilibrium quasiparticles with complex spectrum $\Omega_{n}$ (the spectrum of tilde conjugated nonequilibrium quasiparticles is given by $-\Omega_{n}^{*}$ ). These operators obey the fermionic anticommutation relations, although $c_{n}^{\dagger}$ and $c_{n}\left(\widetilde{c}_{n}^{\dagger}\right.$ and $\left.\widetilde{c}_{n}\right)$ are not Hermitian conjugated to each other.

To find the internal structure and energy spectrum of nonequilibrium quasiparticles we use the equation-of-motion method: If the Liouvillian is diagonal (47), then creation and annihilation operators $c_{n}$ and $c_{n}^{\dagger}$ must satisfy the following equations of motion

$$
\begin{gathered}
{\left[c_{n}^{\dagger}, L^{(0)}\right]=-\Omega_{n} c_{n}^{\dagger},} \\
{\left[c_{n}, L^{(0)}\right]=\Omega_{n} c_{n} .}
\end{gathered}
$$

Equations for $\widetilde{c}_{n}, \widetilde{c}_{n}^{\dagger}$ are obtained from (48|49]) by the tilde conjugation rule. We want to emphasize here that, since $c_{n} \neq\left(c_{n}^{\dagger}\right)^{\dagger}$ and $\Omega_{n}$ is complex, equations (48/49) can not be obtained from each other by the Hermitian conjugation. To avoid unnecessary complication, we do not give a general solution of Eqs. (48, 49), we would rather solve these equations for some particular examples in the next section. Here, we only note that operators which diagonalize the quadratic part of (45) have the following form

$$
\begin{aligned}
& c_{n}^{\dagger}=\sum_{s} \psi_{n, s} b_{s}^{\dagger}+\sum_{k \alpha} \psi_{n, k \alpha} b_{k \alpha}^{\dagger}, \\
& c_{n}=\sum_{s}\left(\psi_{n, s} b_{s}+i \varphi_{n, s} \widetilde{b}_{s}^{\dagger}\right)+\sum_{k \alpha}\left(\psi_{n, k \alpha} b_{k \alpha}+i \varphi_{n, k \alpha} \widetilde{b}_{k \alpha}^{\dagger}\right),
\end{aligned}
$$


and $\widetilde{c}_{n}^{\dagger}$ and $\widetilde{c}_{n}$ are obtained from (150) by the tilde conjugation rule. Note that since the left vacuum $\langle I|$ is the vacuum for $b^{\dagger}, \widetilde{b}^{\dagger}$ operators, nonequilibrium quasiparticle creation operators $c_{n}^{\dagger}, \widetilde{c}_{n}^{\dagger}$ are linear combinations of creation operators only. With help of the anticommutation relations between nonequilibrium quasiparticle creation and annihilation operators, $\left\{c_{n}, c_{n^{\prime}}^{\dagger}\right\}=\delta_{n n^{\prime}},\left\{c_{n}, \widetilde{c}_{n^{\prime}}\right\}=0$, we find that the amplitudes $\psi, \varphi$ satisfy the following orthogonality conditions:

$$
\begin{aligned}
& \sum_{s} \psi_{n, s} \psi_{n^{\prime}, s}+\sum_{k \alpha} \psi_{n, k \alpha} \psi_{n^{\prime}, k \alpha}=\delta_{n n^{\prime}}, \\
& \sum_{s}\left(\psi_{n, s} \varphi_{n^{\prime}, s}^{*}-\varphi_{n, s} \psi_{n^{\prime}, s}^{*}\right)+\sum_{k \alpha}\left(\psi_{n, k \alpha} \varphi_{n^{\prime}, k \alpha}^{*}-\varphi_{n, k \alpha} \psi_{n^{\prime}, k \alpha}^{*}\right)=0 .
\end{aligned}
$$

By diagonalizing the Liouvillian we simultaneously find the nonequilibrium steady state $L^{(0)}\left|\rho_{\infty}^{(0)}\right\rangle=0$ as a (right) vacuum for operators $c_{n}$ and $\widetilde{c}_{n}$. Using the transformation inverse to (50) we can express any operator in terms of nonequilibrium quasiparticle operators. Then all physical quantities are calculated as an expectation value with respect to $\langle I|$ and $\left|\rho_{\infty}^{(0)}\right\rangle$ vacuum states (see Eq. (7)) in the nonequilibrium Hartree-Fock approximation. For the steady state current from the lead $\alpha=L, R$

$$
J_{\alpha}^{(0)}=-\frac{d}{d t} \sum_{k}\left\langle I\left|a_{k \alpha}^{\dagger} a_{k \alpha}\right| \rho_{\infty}^{(0)}\right\rangle=-i \sum_{k s} t_{s k \alpha}\left\langle I\left|\left(a_{k \alpha}^{\dagger} a_{s}-a_{s}^{\dagger} a_{k \alpha}\right)\right| \rho_{\infty}^{(0)}\right\rangle
$$

we derive, after some algebra, that

$$
J_{\alpha}^{(0)}=-i \sum_{k s n} T_{s k \alpha}\left(\psi_{n, k \alpha}^{*} \varphi_{n, s}^{*}-\psi_{n, s}^{*} \varphi_{n, k \alpha}^{*}\right)=-2 \operatorname{Im} \sum_{k s n} T_{s k \alpha} \psi_{n, k \alpha} \varphi_{n, s}
$$

where the last equality follows from

$$
\left[b_{s}, \widetilde{b}_{k \alpha}\right]=\sum_{n}\left(\psi_{n, s} \varphi_{n, k \alpha}-\psi_{n, k \alpha}^{*} \varphi_{n, s}^{*}\right)=0 .
$$

For the steady state single-particle density matrix we have

$$
n_{s_{2} s_{1}}=\sum_{s_{1}^{\prime}, s_{2}^{\prime}, n} D_{s_{1} s_{1}^{\prime}} D_{s_{2} s_{2}^{\prime}} \psi_{n, s_{1}^{\prime}} \varphi_{n, s_{2}^{\prime}} .
$$

Let us summarize the main results of this section. We have shown that the quadratic part of the Liouvillian (28) can be diagonalized by three canonical transformations. The first transformation diagonalizes the reservoir part of the Liouvillian including dissipator, the second is performed on the electrons in the interacting region and diagonalizes the Hartree-Fock part of $L_{S}$, and the last transformation mixes operators from the interacting region and the leads and diagonalizes the entire quadratic part of the Liouvillian. Two of these three transformations are not unitary, but all of them are canonical, which means that the anticommutation relations between particle creation and annihilation operators are preserved. The remaining part $L_{S}^{\prime}$ can be taken into account via standard perturbation theory or nonperturbatively by, for example, coupled cluster method ${ }^{37}$ or configuration interaction theory $\underline{\underline{38}}$ In fact, any other method for the correlated electronic structure calculations can be extended to nonequilibrium within our approach, although special care should be taken because one has to work with the nonunitary representation of the creation and annihilation operators.

\section{ANALYTICAL AND NUMERICAL EXAMPLES}

\section{A. Transport through a single-level molecule}

To illustrate the theory we consider a single-level molecule connected to two leads held at different chemical potentials. The molecule is described by the Hamiltonian

$$
H_{S}=\varepsilon a^{\dagger} a .
$$

In this section we obtain nonequilibrium steady state wavefunction $\left|\rho_{\infty}\right\rangle$ for this model and compare the results with the Landauer theory. To simplify the notation we assume throughout the calculations that the matrix elements of 
the tunneling interaction are real and do not depend on the leads energy levels, i.e., $t_{k \alpha}=t_{k \alpha}^{*}=t$. So the Liouvillian becomes:

$$
L=\varepsilon\left(a^{\dagger} a-\widetilde{a}^{\dagger} \widetilde{a}\right)-t \sum_{k \alpha}\left(a_{k \alpha}^{\dagger} a-\widetilde{a}_{k \alpha}^{\dagger} \widetilde{a}+h . c .\right)+\sum_{k \alpha} \varepsilon_{k \alpha}\left(a_{k \alpha}^{\dagger} a_{k \alpha}-\widetilde{a}_{k \alpha}^{\dagger} \widetilde{a}_{k \alpha}\right)-i \sum_{k \alpha} \Pi_{k \alpha},
$$

where $\Pi_{k \alpha}$ is the dissipator for the lead $\alpha=L, R$ taken in the standard form (32). Our goal now is the exact diagonalization of the Liouvillian (57) in terms of nonequilibrium, non-Hermitian quasiparticles:

$$
L=\sum_{n=1}^{2 N+1}\left(\Omega_{n} c_{n}^{\dagger} c_{n}-\Omega_{n}^{*} \widetilde{c}_{n}^{\dagger} \widetilde{c}_{n}\right)
$$

To do this, we first perform the transformations (35) over the leads operators and $b^{\dagger}=a^{\dagger}-i \widetilde{a}, b=a$ for the molecular level. Then the equations of motion (48) give the following structure for the nonequilibrium quasiparticle creation and annihilation operators:

$$
\begin{gathered}
c_{n}^{\dagger}=\psi_{n} b^{\dagger}+\sum_{k \alpha} \psi_{n, k \alpha} b_{k \alpha}^{\dagger}, \\
c_{n}=\psi_{n} b+i \varphi_{n} \widetilde{b}^{\dagger}+\sum_{k \alpha}\left(\psi_{n, k \alpha} b_{k \alpha}+i \varphi_{n, k \alpha} \widetilde{b}_{k \alpha}^{\dagger}\right) .
\end{gathered}
$$

The amplitudes $\psi_{n}, \psi_{n, k \alpha}$ and quasiparticle energies $\Omega_{n}$ are the solution of the following eigenvalue problems

$$
\begin{aligned}
& \varepsilon \psi_{n}-t \sum_{k \alpha} \psi_{n, k \alpha}=\Omega_{n} \psi_{n}, \\
& E_{k \alpha} \psi_{n, k \alpha}-t \psi_{n}=\Omega_{n} \psi_{n, k \alpha},
\end{aligned}
$$

whereas the amplitudes $\varphi_{n}$ and $\varphi_{n, k \alpha}$ satisfy the following nonhomogeneous system of linear equations:

$$
\begin{aligned}
& \left(\varepsilon-\Omega_{n}\right) \varphi_{n}-t \sum_{k \alpha} \varphi_{n, k \alpha}=t \sum_{k \alpha} f_{k \alpha} \psi_{n, k \alpha}, \\
& \left(E_{k \alpha}^{*}-\Omega_{n}\right) \varphi_{n, k \alpha}-t \varphi_{n}=-t f_{k \alpha} \psi_{n} .
\end{aligned}
$$

The amplitudes $\psi_{n}, \psi_{n, k a}$ should be normalized according to the first equation in (51). Operators $\widetilde{c}_{n}, \widetilde{c}_{n}^{\dagger}$ are obtained from (59) and (60) by the tilde conjugation. Again, the transformations (59]60) are canonical but nonunitary, so $\left\{c_{n}, c_{n^{\prime}}^{\dagger}\right\}=\delta_{n n^{\prime}}$ and $\left(c_{n}\right)^{\dagger} \neq c_{n}^{\dagger}$, and nontilde and tilde operators anticommute. Although the analytical expressions for quasiparticle amplitudes and spectrum can be obtained (see Appendix), we found out that it is more convenient for practical calculations and for application to more complex system to solve Eqs. (61, 62) numerically. Namely, we first solve the eigenvalue problem (61), and then with the known quasiparticle spectrum $\Omega_{n}$ and amplitudes $\psi_{n}, \psi_{n, k \alpha}$ we solve the linear system of equations (62).

Since the Liouvillian (57) is diagonal in terms of $c_{n}^{\dagger}, c_{n}$ and their tilde conjugate $\widetilde{c}_{n}^{\dagger}, \widetilde{c}_{n}$, we can associate the vacuum of $c_{n}, \widetilde{c}_{n}$ with the nonequilibrium steady state:

$$
c_{n}\left|\rho_{\infty}\right\rangle=\widetilde{c}_{n}\left|\rho_{\infty}\right\rangle=0 .
$$

By the construction of $c_{n}^{\dagger}, \widetilde{c}_{n}^{\dagger},\langle I| c_{n}^{\dagger}=\langle I| \widetilde{c}_{n}^{\dagger}=0$.

Using the transformation inverse to (59, 60) (see Appendix) we calculate the steady state current and nonequilibrium electron populations of the molecule and leads levels. For the current, according to (53), we get

$$
J_{\alpha}=-2 t \operatorname{Im} \sum_{k n} \psi_{n, k \alpha} \varphi_{n}
$$

while for the population of the molecule and leads levels we derive

$$
\begin{aligned}
& \left\langle a^{\dagger} a\right\rangle=\left\langle I\left|a^{\dagger} a\right| \rho_{\infty}\right\rangle=\sum_{n} \psi_{n} \varphi_{n}, \\
& \left\langle a_{k \alpha}^{\dagger} a_{k \alpha}\right\rangle=\left\langle I\left|a_{k \alpha}^{\dagger} a_{k \alpha}\right| \rho_{\infty}\right\rangle=f_{k \alpha}+\sum_{n} \psi_{n, k \alpha} \varphi_{n, k \alpha} .
\end{aligned}
$$



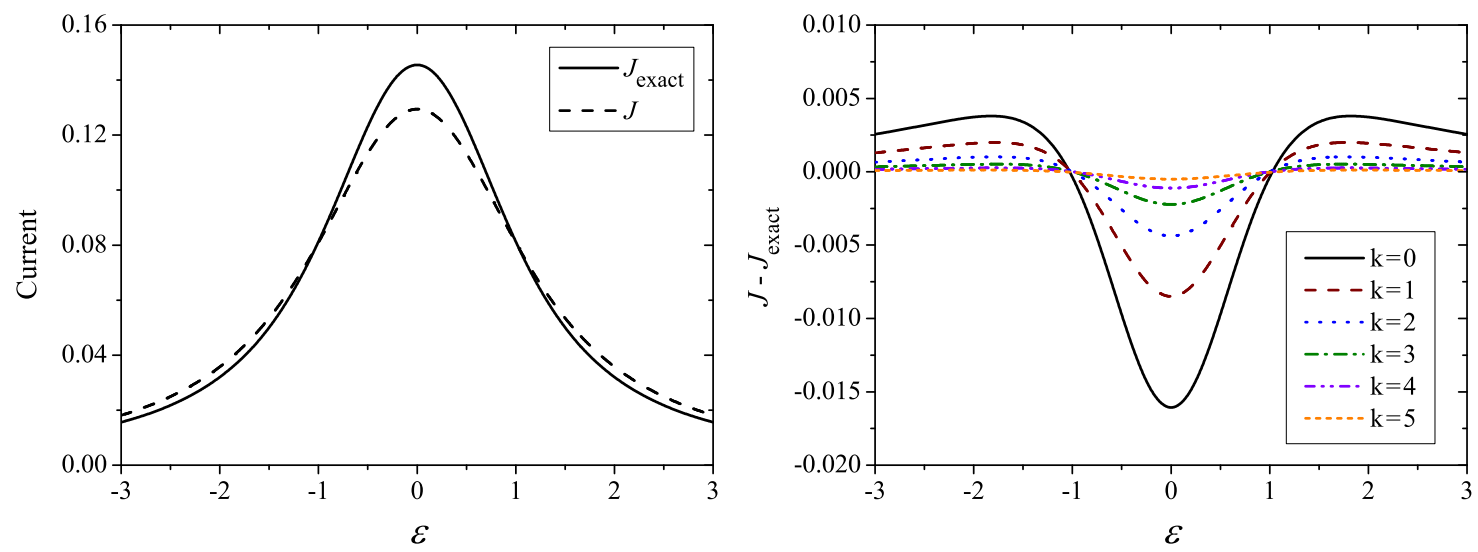

FIG. 2: (Color) Left panel: The current through the one-level molecule as a function of the energy of the level. We choose chemical potentials of the leads to be $\mu_{L, R}= \pm 0.5$ and $T_{L, R}=0.1$. Right panel: The difference between calculated and exact currents for different values of $N=100 \times 2^{k}$.
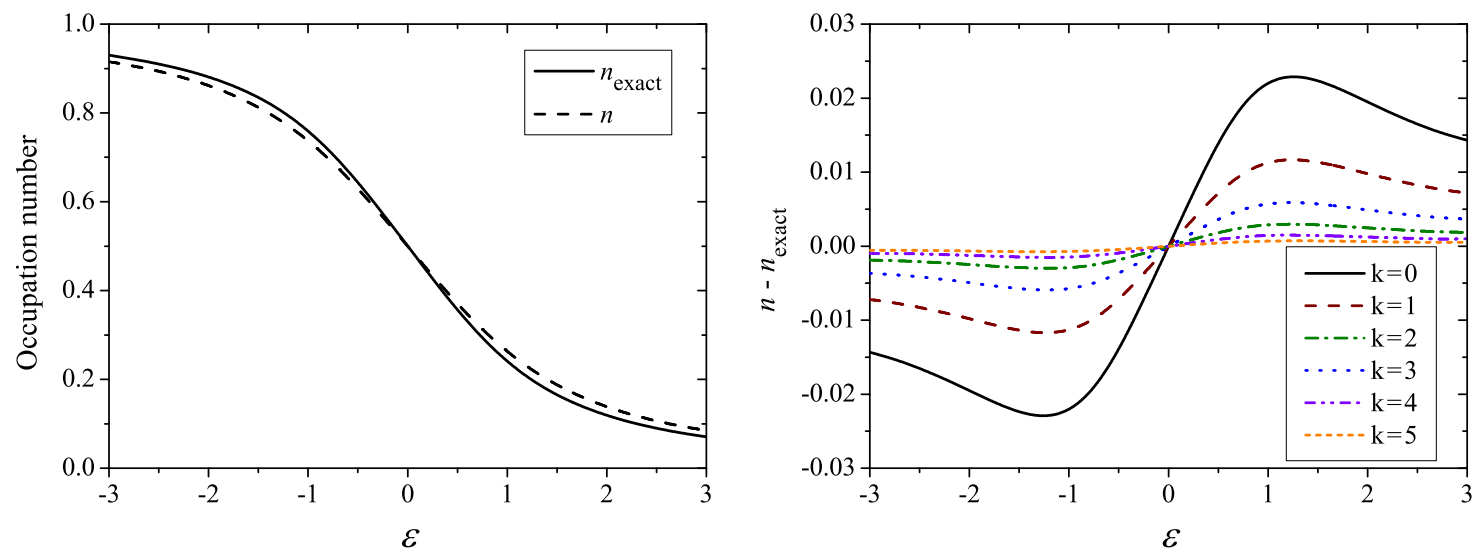

FIG. 3: (Color) Left panel: The occupation number [Eq. (65)] for the one-level molecule as a function of energy of the level. Right panel: The difference between calculated and exact occupation numbers for different values of $N$. The parameters are the same as in Fig. 2

As is expected, these occupation numbers are real (see Appendix). Moreover, using the explicit analytical expressions for amplitudes $\psi_{n, k \alpha}, \varphi_{n, k \alpha}$ it can be demonstrated that the total number of electrons in both the leads is conserved quantity, i.e.,

$$
\sum_{k \alpha}\left\langle a_{k \alpha}^{\dagger} a_{k \alpha}\right\rangle=\sum_{k \alpha} f_{k \alpha}
$$

In what follows we consider the case of identical left and right leads, i.e., $E_{k L}=E_{k R}=E_{k}$. Then $N$ eigenstates of (61) coincide with the energies of the leads' levels, i.e., $\Omega_{n}=E_{l}$. Using analytical expressions for the amplitudes $\psi_{n, k \alpha}$ and $\varphi_{n}$ (see Appendix) it can be easily shown that only eigenstates with $\Omega_{n}=E_{l}$ contribute the sum over $n$ in (64). (This result is also valid when $t_{R}=\alpha t_{L}$.) It is similar to Meir and Wingreen observation that the expression for the current can be simplified within NEGF formalism when the spectral function of the coupling to the left lead is proportional to the spectral function of the couplings to the right lead. $\frac{62}{2}$ On the contrary, eigenstates with $\Omega_{n}=E_{l}$ do not contribute to the molecule population in (65).

Let us compare the results obtained within our approach with the results given by the Landauer theory $\underline{\underline{63}}$ The Landauer theory is exact for noninteracting electrons and it gives the following expression for the current $\underline{63}$

$$
J_{\text {exact }}=\frac{1}{2 \pi} \int d \omega \frac{\Gamma^{2}(\omega)\left(f_{L}(\omega)-f_{R}(\omega)\right)}{(\omega-\varepsilon-2 \Lambda(\omega))^{2}+(\Gamma(\omega))^{2}},
$$


and for the population

$$
n_{\text {exact }}=\frac{1}{2 \pi} \int d \omega \frac{\Gamma(\omega)\left(f_{L}(\omega)+f_{R}(\omega)\right)}{(\omega-\varepsilon-2 \Lambda(\omega))^{2}+(\Gamma(\omega))^{2}} .
$$

Here $\Lambda(\omega)$ and $\Gamma(\omega)$ are real and imaginary parts of the self-energy of the leads

$$
\Sigma(\omega)=t^{2} \sum_{k} \frac{1}{\omega-\varepsilon_{k \alpha}+i \gamma}=\Lambda(\omega)-\frac{i}{2} \Gamma(\omega) .
$$

Notice that the self-energy (69) exactly appears in secular equation (A3). We do not distinguish between the selfenergies of the left and right leads, since the both leads are identical. In numerical calculations by the Landauer theory we put $N \rightarrow \infty, \gamma \rightarrow 0$ and assume that leads densities of states are constant within the energy bandwidth $\left[E_{\min }: E_{\max }\right]$. Then $\Gamma(\omega)=\Gamma$ for $E_{\min } \leq \omega \leq E_{\max }$ and zero otherwise. For $\Lambda(\omega)$ we have the Cauchy principal value integral

$$
\Lambda(\omega)=\frac{1}{2 \pi} \mathrm{P} \int_{-\infty}^{\infty} \frac{\Gamma(\varepsilon) d \varepsilon}{\omega-\varepsilon}=\frac{\Gamma}{2 \pi} \ln \left|\frac{\omega-E_{\min }}{\omega-E_{\max }}\right|
$$

In our calculations with the kinetic equation we assume that $N$ energy levels in each lead are evenly spaced in the bandwidth $\left[E_{\min }: E_{\max }\right]=[-5: 5]$. The tunneling coupling strength $t$ is computed from the $\Gamma=2 \pi \eta t^{2}=1$, where $\eta=N /\left(E_{\max }-E_{\min }\right)$ is the density of states. Below we put $\gamma=2 \Delta \varepsilon$, where $\Delta \varepsilon$ is the energy spacing between states in the leads. Under this choice of the parameters $\Gamma(\omega)$, as is computed by (69), is constant equal to 1 within the bandwidth of the leads and vanishes when $\omega$ is outside the bandwidth.

In the left panel of Fig. 2, we plot the current [Eq. (64)] calculated within our approach for $N=100$ as a function of the level energy along with the exact current computed by the Landauer formula (67). We see that the calculated current agrees well with the exact one. The largest deviation between the two currents is obtained when the currents reach their maxima. In the right panel of Fig. 2 we plot the difference between the calculated within our approach and exact currents for different values of $N$. It is evident from the figure that the difference becomes smaller as the leads densities of states increase. It agrees with our observation (see Appendix) that our expression for the current (64) becomes the Landauer formula (67) in the limit of macroscopically large leads. We also calculate nonequilibrium population of the molecule and compare them with the exact values (68) for different values of $N$. The results are shown in Fig. 3. Again, we reproduce the exact results by increasing the part of the leads included into the consideration.

\section{B. Out of equilibrium Anderson model in Hartree-Fock approximation}

In this section, we apply our method to a physically more interesting example, namely to electron transport through a spin-degenerate single level with local Coulomb interaction (so called Anderson model). The Hamiltonian for the molecule has the form

$$
H_{S}=\varepsilon \sum_{\sigma} a_{\sigma}^{\dagger} a_{\sigma}+U a_{\uparrow}^{\dagger} a_{\uparrow} a_{\downarrow}^{\dagger} a_{\downarrow}
$$

where $a_{\sigma}^{\dagger}, a_{\sigma}$ are the creation and annihilation operators for the spin-up $(\sigma=\uparrow)$ and spin-down $(\sigma=\downarrow)$ electrons in the molecule, $\varepsilon$ is the energy of the single level in the molecule, and the charging energy $U$ characterizes the Coulomb interaction of electrons in the molecule. Here we restrict our consideration by a nonmagnetic system, therefore the energy levels in the leads are spin degenerates and all the coupling strengths $t_{k \alpha \sigma}$ take the same value $t$. Under these assumptions we have

$$
H_{B}+H_{T}=\sum_{k \alpha \sigma} \varepsilon_{k \alpha} a_{k \alpha \sigma}^{\dagger} a_{k \alpha \sigma}-t \sum_{k \alpha \sigma}\left(a_{k \alpha \sigma}^{\dagger} a_{\sigma}+\text { h.c. }\right) .
$$

We are interested in quantum transport in Coulomb blockade regime, where we charging energy $U$ is much greater than the effective coupling $\Gamma_{\uparrow, \downarrow}=2 \pi \eta t^{2}$ between the molecule and the leads. So we work in the regime of strong Coulomb interaction and weak coupling to the leads. 

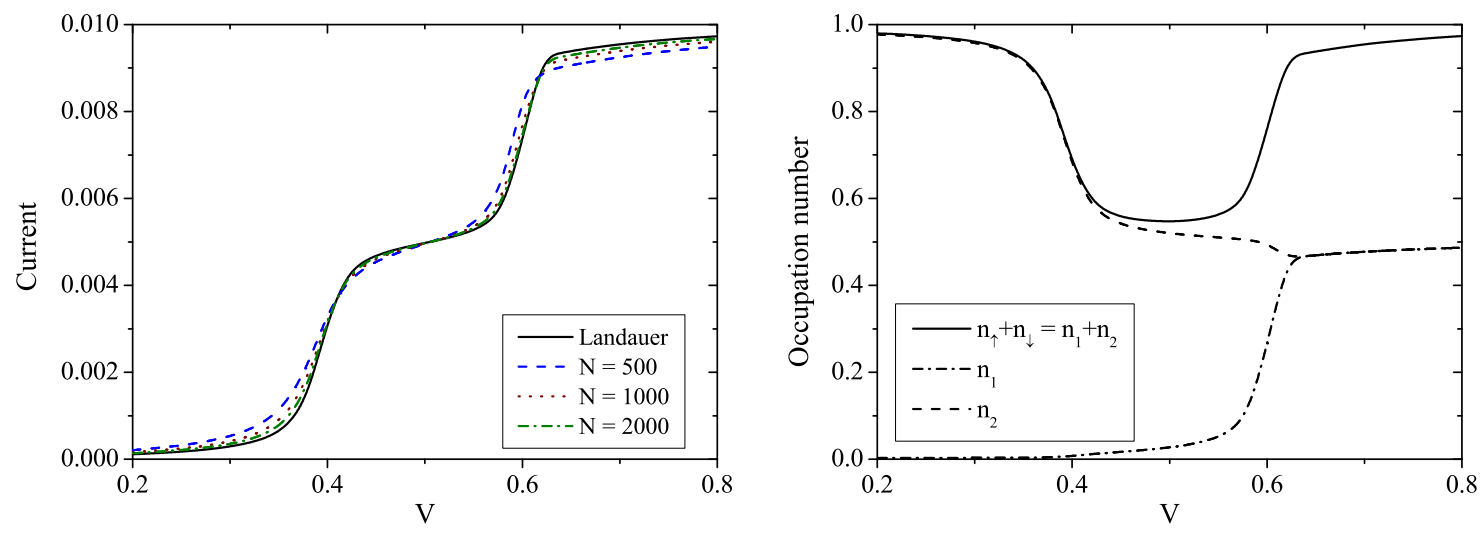

FIG. 4: (Color) The current (left panel) and the occupation numbers (right panel) for out of equilibrium Anderson model at fixed gate voltage $V_{g}=-0.2$ as a function of voltage difference between left and right leads. For the current we show the results obtained with different number of states $N$ in each lead and the exact Hartree-Fock result. In the right panel the total occupation number and the nonequilibrium populations of the Hartree-Fock levels are shown for $N=2000$.

Using the Wick theorem with respect to nonequilibrium steady state vacuum $\left|\rho_{\infty}^{(0)}\right\rangle$ (which is yet to be defined) from the right and the left vacuum $\langle I|$, we obtain the Hartree-Fock part of $H_{S}$ in the following form

$$
H_{S}^{0}=\sum_{\sigma}\left(\varepsilon+U n_{-\sigma}\right) a_{\sigma}^{\dagger} a_{\sigma}-U\left(n_{\downarrow \uparrow} a_{\downarrow}^{\dagger} a_{\uparrow}+n_{\uparrow \downarrow} a_{\uparrow}^{\dagger} a_{\downarrow}\right)
$$

where $n_{\sigma}=\left\langle I\left|a_{\sigma}^{\dagger} a_{\sigma}\right| \rho_{\infty}^{(0)}\right\rangle$ and $n_{\sigma \sigma^{\prime}}=\left\langle I\left|a_{\sigma^{\prime}}^{\dagger} a_{\sigma}\right| \rho_{\infty}^{(0)}\right\rangle$. Since the system is nonmagnetic, the occupation numbers for both spin orientations are the same $n_{\sigma}=n_{-\sigma}$. Moreover, due to the symmetry reasons $n_{\uparrow \downarrow}=n_{\downarrow \uparrow}$. Then the following unitary transformation

$$
\alpha_{1}^{\dagger}=\frac{1}{\sqrt{2}}\left(a_{\uparrow}^{\dagger}-a_{\downarrow}^{\dagger}\right), \quad \alpha_{2}^{\dagger}=\frac{1}{\sqrt{2}}\left(a_{\uparrow}^{\dagger}+a_{\downarrow}^{\dagger}\right)
$$

brings $H_{S}^{0}$ to a diagonal form

$$
H_{S}^{0}=\sum_{s=1,2} \varepsilon_{s} \alpha_{s}^{\dagger} \alpha_{s}
$$

where the Hartree-Fock mean-field energies are

$$
\varepsilon_{1(2)}=\varepsilon+U\left(n_{\sigma} \pm n_{\uparrow \downarrow}\right)=\varepsilon+U n_{2(1)},
$$

and $n_{s}=\left\langle I\left|\alpha_{s}^{\dagger} \alpha_{s}\right| \rho_{\infty}^{(0)}\right\rangle$ is the occupation number for the Hartree-Fock single-particle level "s" (the crossover matrix element $\left\langle I\left|\alpha_{s}^{\dagger} \alpha_{s^{\prime} \neq s}\right| \rho_{\infty}^{(0)}\right\rangle$ is equal zero) and $s=1(2)$ corresponds to " $+(-)$ " signs respectively. We can regard $\alpha_{1}^{\dagger}$ as the antibonding orbital creation operator, and $\alpha_{2}^{\dagger}$ as the creation operator for the bonding orbital in the dot. From the above equation it follows that if $n_{\uparrow \downarrow} \neq 0$, the Hartree-Fock bonding/antibonding energies $\varepsilon_{1(2)}$ are split and the populations of bonding/antibonding orbitals are generally not the same $n_{1} \neq n_{2}$.

Following the procedure described in Sect. III we introduce $b_{s}^{\dagger}$ and $b_{k \alpha \sigma}^{\dagger}$ operators which annihilate the left vacuum $\langle I|$. Then the quadratic part of the Liouvillian becomes

$$
\begin{aligned}
L^{(0)}= & \sum_{s} \varepsilon_{s}\left(b_{s}^{\dagger} b_{s}-\widetilde{b}_{s}^{\dagger} \widetilde{b}_{s}\right)+L_{B} \\
& -\sum_{k \alpha \sigma s} T_{s \sigma}\left\{\left(b_{k \alpha \sigma}^{\dagger} b_{s}+b_{s}^{\dagger} b_{k \alpha \sigma}\right)-\left(\widetilde{b}_{k \alpha \sigma}^{\dagger} \widetilde{b}_{s}+\widetilde{b}_{s}^{\dagger} \widetilde{b}_{k \alpha \sigma}\right)+i f_{k \alpha}\left(b_{k \alpha \sigma}^{\dagger} \widetilde{b}_{s}^{\dagger}+\widetilde{b}_{k \alpha \sigma}^{\dagger} b_{s}^{\dagger}\right)\right\},
\end{aligned}
$$

where $T_{1 \downarrow}=-t / \sqrt{2}$ and all other $T_{s \sigma}$ are equal $t / \sqrt{2}$. Likewise the noninteracting single-level model considered in the previous section, the Liouvillian (77) can be diagonalized in terms of nonequilibrium quasiparticles by the 

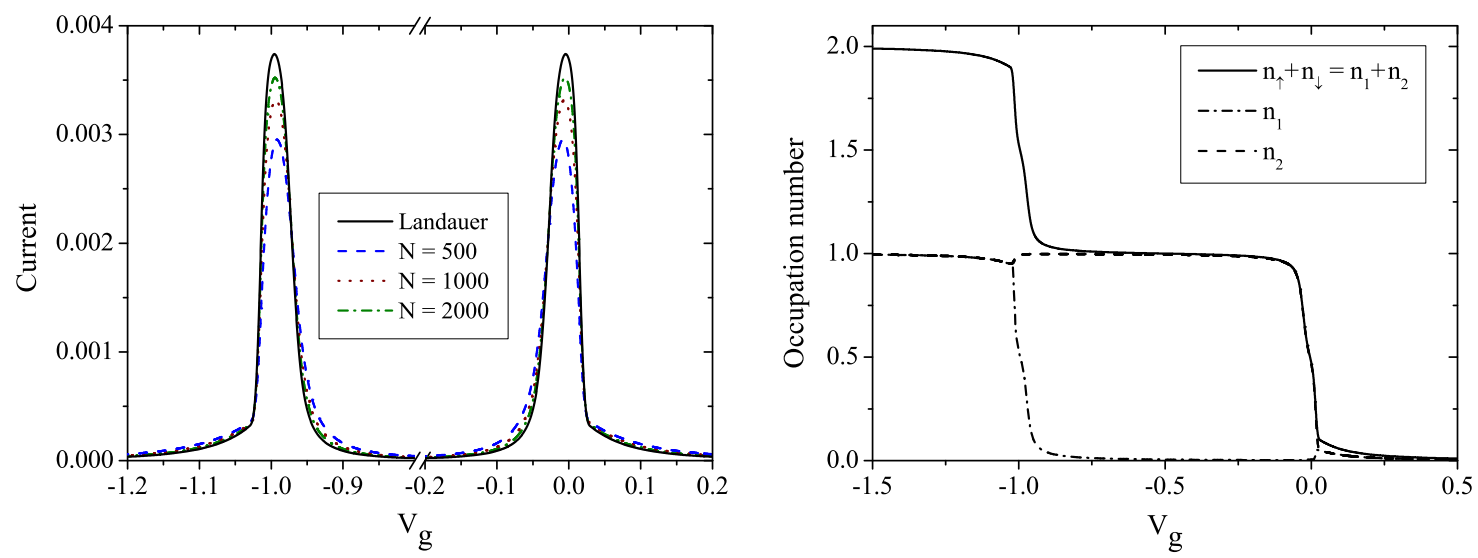

FIG. 5: (Color) The current (left panel) and the occupation number (right panel) for out of equilibrium Anderson model at small applied voltage $\mu_{L, R}= \pm 0.025$ as a function of gate voltage. The parameters and notations are the same as in Fig. 4 .

equation of motion method. The structure of nonequilibrium quasiparticles, which diagonalize (77) is the following $(n=1, \ldots, 2+4 N)$

$$
\begin{aligned}
& c_{n}^{\dagger}=\sum_{s} \psi_{n, s} b_{s}^{\dagger}+\sum_{k \alpha \sigma} \psi_{n, k \alpha \sigma} b_{k \alpha \sigma}^{\dagger}, \quad \widetilde{c}_{n}^{\dagger}=\left(c_{n}^{\dagger}\right), \\
& c_{n}=\sum_{s}\left(\psi_{n, s} b_{s}+i \varphi_{n, s} \widetilde{b}_{s}^{\dagger}\right)+\sum_{k \alpha \sigma}\left(\psi_{n, k \alpha \sigma} b_{k \alpha \sigma}+i \varphi_{n, k \alpha \sigma} \widetilde{b}_{k \alpha \sigma}^{\dagger}\right), \quad \widetilde{c}_{n}=\left(c_{n}\right),
\end{aligned}
$$

where the amplitudes $\psi, \varphi$ and the spectrum $\Omega_{n}$ are obey of the equations $(s=1,2)$

$$
\begin{aligned}
& \varepsilon_{s} \psi_{n, s}-\sum_{k \alpha \sigma} T_{s \sigma} \psi_{n, k \alpha \sigma}=\Omega_{n} \psi_{n, s}, \\
& E_{k \alpha} \psi_{n, k \alpha \sigma}-\sum_{s} T_{s \sigma} \psi_{n, s}=\Omega_{n} \psi_{n, k \alpha \sigma},
\end{aligned}
$$

and

$$
\begin{aligned}
& \left(\varepsilon_{s}-\Omega_{n}\right) \varphi_{n, s}-\sum_{k \alpha \sigma} T_{s \sigma} \varphi_{n, k \alpha \sigma}=\sum_{k \alpha} t_{s \sigma} f_{k \alpha} \psi_{n, k \alpha}, \\
& \left(E_{k \alpha}^{*}-\Omega_{n}\right) \varphi_{n, k \alpha \sigma}-\sum_{s} T_{s \sigma} \varphi_{n, s}=-f_{k \alpha} \sum_{s} t_{s \sigma} \psi_{n, s} .
\end{aligned}
$$

The amplitudes depend on Hartree-Fock occupations numbers $n_{s}$, which in turn depend on the amplitudes:

$$
n_{s}=\sum_{n} \psi_{n, s} \varphi_{n, s}
$$

Therefore the equations (79|80) should be solved via self-consistent iterations. After the diagonalization, the Liouvillian (77) takes the form (47). Having found the amplitudes for the nonequilibrium quasiparticles we can compute the nonequilibrium population of the molecule $\left(n_{\uparrow}+n_{\downarrow}=n_{1}+n_{2}\right)$ as well as the steady state current (53).

Likewise the example with single molecule level, in case of identical leads the system (79) have $N$ twice degenerate eigenstates equal to the energy levels in the leads. Only these eigenstates contribute to the steady state current, but they do not contribute to the population of the molecule.

In our further numerical calculations we assume the symmetrical voltage drop, $\mu_{L, R}=E_{F} \pm \frac{1}{2} V$, and $E_{F}=0$. Also, we introduce gate voltage $V_{g}$, so that the energy of molecular level is $\varepsilon\left(V_{g}\right)=E_{F}+V_{g}$. We put $U=1.0$ for the strength of the Coulomb interaction. The leads energy levels are spaced in the bandwidth $[-1: 1]$ and $\Gamma_{\uparrow, \downarrow}=0.01$, $T_{L, R}=0.001$.

Fig. 4 shows the calculated current through the molecule and the occupation numbers as function of the bias voltage applied across the junction at fixed gate voltage $V_{g}=-0.2$. While both chemical potentials are above the molecular energy level $\varepsilon=-0.2$ the electric current through the molecule is small and the average occupation number 
of the molecule is close to unity. It means that the bonding Hartree-Fock level $s=2$ is below $\mu_{L, R}\left(\varepsilon_{2}=\varepsilon\right)$ and it is totally occupied $\left(n_{2}=1\right)$ while the antibonding level $s=1$ is above $\mu_{L, R}\left(\varepsilon_{1}=\varepsilon+U\right)$ and it is empty $\left(n_{1}=0\right)$. The first step in the current occurs when the right chemical potential reaches the energy $\varepsilon(V \approx 0.4)$ and the Hartree-Fock bonding level $s=2$ becomes involved in electronic transport. As a result the occupation of this level decreases to 0.5 , and the energy of the empty Hartree-Fock level $s=1$ reduces to $\varepsilon_{1}=\varepsilon+0.5 U$. The total number of electrons is the molecule becomes $n_{1}+n_{2}=0.5$. The second step in the current occurs when the left chemical potential reaches the energy level $\varepsilon_{1}=\varepsilon+0.5 U(V \approx 0.6)$ for the antibonding orbital and electrons starts to flow through it. This increases the occupation number of the antibonding Hartree-Fock orbital level $s=1$ from zero to 0.5 and, therefore, the bonding level $s=2$ is pushed up from $\varepsilon_{2}=\varepsilon$ to $\varepsilon_{2}=\varepsilon+0.5 U$. At higher voltage $(V>0.6)$ the Hartree-Fock levels become degenerate and they both lay between $\mu_{L}$ and $\mu_{R}$. Since each of them is half-occupied, the total number of electrons in the molecule becomes again equal to unity.

Fig. 5] shows the current and the occupation numbers as a function of the gate voltage $V_{g}$, at small applied voltage $\mu_{L, R}= \pm 0.025$. At $V_{g}<-1.0$ both Hartree-Fock levels are below $\left(\varepsilon_{1,2}=\varepsilon+U<0\right)$ the chemical potentials for both leads. Accordingly, the occupation number is equal to two and the current through the junction is close to zero. At $V_{g} \approx-1.0$ the molecule starts to conduct electrons This result in a pronounced increase in the current accompanied by a decrease in the occupation number. At $-1.0<V_{g}<0$ the Hartree-Fock bonding level is below chemical potentials $\left(\varepsilon_{2}=\varepsilon\right)$, and antibonding level is above them $\left(\varepsilon_{1}=\varepsilon+U\right)$. Therefore the occupation number is equal to unity and the current through the molecule becomes almost zero At $V_{g} \approx 0.0$ the bonding level becomes involved in the electronic transport giving rise to the current increase. At higher gate voltage, when the single-particle level $\varepsilon$ is above the chemical potentials, the molecule become empty and the current again drops to zero.

In Figs. 4 and 5] we compare our results with the exact Landauer current through Hartree-Fock levels. To obtain the exact current we first solve iteratively the nonlinear equations $n_{1(2)}=n_{\text {exact }}\left(\varepsilon_{1(2)}\right)$ (68). Then the Hartree-Fock levels $\varepsilon_{1(2)}$ are used in (67) to compute the current. Figs. 4 and 5 show that the currents calculated within the present approach converge to the exact result with increasing value of $N$.

\section{CONCLUSIONS}

Based on the super-fermion representation of the Liouville space, we developed an approach which enables us to transform the quantum master equations to the super-Fock space and then to use standard methods of quantum field theory to solve them in the nonequilibrium steady state regime. We worked with the Lindblad master equation in this paper although the derivations can be readily extended to more sophisticated master equations. The main technical difficulty of the approach is that the left vacuum is always different from the right vacuum state unless we have a system in thermodynamic equilibrium. This prohibits the use of the unitary transformations to diagonalize the Liouvillian. The problem was circumvented by the development of a set of nonunitary, canonical transformations between particle creation and annihilation operators. These nonunitary canonical transformations preserve the anticommutation relations between the fermionic operators and significantly facilitate the derivations.

Starting with Lindblad master equation for the electron transport through the interacting region, we converted the problem of finding the nonequilibrium steady state to the many-body problem with non-Hermitian "Hamiltonian" in super-Fock space. Then we demonstrated that despite the fact that the left vacuum is different from the right vacuum we still can use the Wick theorem. Using the Wick theorem we transformed the Liouvillian to the normal ordered form, introduced nonequilibrium quasiparticles and developed a general many-body theory for electron transport through interacting region. We applied the approach to electron transport through a single level molecule. Then we considered the system with electron-electron interactions, namely out of equilibrium Anderson model in Hartree-Fock approximation. The Wick theorem was applied to obtain the Hartree-Fock solution of the transport problem. We demonstrated that it is consistent with the Landauer theory, which is exact for these models. Being formulated in the language of Fock spaces, creation and annihilation operators and normal ordered "Hamiltonian" the proposed approach is not only capable of doing perturbative calculations but also has a great complementarity to the nonperturbative many-body methods of molecular electronic structure calculations such as, for example, coupled cluster or configuration interaction theories.

\section{Acknowledgments}

The authors thank M. Esposito, M. Gelin, and T. Prosen for valuable discussions. This work has been supported by the Francqui Foundation and by the Belgian Federal Government under the Inter-university Attraction Pole project NOSY P06/02. 


\section{Appendix A: Structure of nonequilibrium quasiparticles creation and annihilation operators for single level} model

For the single level model the analytical solution of Eqs. (61, 62) has the following form:

$$
\begin{aligned}
& \psi_{n}=\mathcal{N}_{n}^{-1 / 2}, \quad \psi_{n, k \alpha}=\frac{t}{E_{k \alpha}-\Omega_{n}} \psi_{n}, \\
& \varphi_{n}=\mathcal{N}_{n}^{-1 / 2} \frac{\sum_{k \alpha} \frac{f_{k \alpha} \gamma_{k \alpha}}{\left(E_{k \alpha}-\Omega_{n}\right)\left(E_{k \alpha}^{*}-\Omega_{n}\right)}}{\sum_{k \alpha} \frac{\gamma_{k \alpha}}{\left(E_{k \alpha}-\Omega_{n}\right)\left(E_{k \alpha}^{*}-\Omega_{n}\right)}}, \quad \varphi_{n, k \alpha}=\frac{t}{E_{k \alpha}^{*}-\Omega_{n}}\left(\varphi_{n}-\psi_{n} f_{k \alpha}\right) .
\end{aligned}
$$

where $\mathcal{N}_{n}=1+t^{2} \sum_{k \alpha}\left(E_{k \alpha}-\Omega_{n}\right)^{-2}$, and $\Omega_{n}$ obey the secular equation

$$
(\varepsilon-\Omega)+t^{2} \sum_{k \alpha} \frac{1}{\Omega-E_{k \alpha}}=0
$$

Some constraints on quasiparticle amplitudes can be obtained by using the transformations inverse to (59]60):

$$
\begin{aligned}
& b^{\dagger}=\sum_{n} \psi_{n} c_{n}^{\dagger}, \quad b=\sum_{n}\left(\psi_{n} c_{n}-i \varphi_{n}^{*} \widetilde{c}_{n}^{\dagger}\right), \\
& b_{k \alpha}^{\dagger}=\sum_{n} \psi_{n, k \alpha} c_{n}^{\dagger}, \quad b_{k \alpha}=\sum_{n}\left(\psi_{n, k \alpha} c_{n}-i \varphi_{n, k \alpha}^{*} \widetilde{c}_{n}^{\dagger}\right)
\end{aligned}
$$

(the expressions for $\widetilde{b}, \widetilde{b}^{\dagger}, \widetilde{b}_{k \alpha}, \widetilde{b}_{k \alpha}^{\dagger}$ are obtained from (A4) by the tilde conjugation). In particular, from $\left\{b_{k \alpha}, \widetilde{b}_{k \alpha}\right\}=0$ and $\{b, \widetilde{b}\}=0$ it follows that $\sum_{n} \psi_{n, k \alpha} \varphi_{n, k \alpha}$ and $\sum_{n} \psi_{n} \varphi_{n}$ are real.

If we have identical left and right leads, i.e., if $E_{k L}=E_{k R}=E_{k}$, then $N$ eigenstates of (61) coincide with the energies of the leads levels, i.e., $\Omega_{n}=E_{l}$. For such normalized eigenstates we have

$$
\psi_{n}=0, \quad \psi_{n, k L}=-\psi_{n, k R}=\delta_{k l} \frac{1}{\sqrt{2}},
$$

where $\delta_{k l}$ is the Kroneker symbol, and

$$
\varphi_{n}=2^{-1 / 2} \frac{t\left(f_{l L}-f_{l R}\right)}{\left(\varepsilon-E_{l}\right)+2 t^{2} \sum_{k} \frac{1}{\left(E_{l}-E_{k}^{*}\right)}}, \quad \varphi_{n, k \alpha}=\frac{t \varphi_{n}}{\left(E_{k}^{*}-E_{l}\right)} .
$$

This result is also valid when $t_{R}=\alpha t_{L}$. The only difference is that $\psi_{n, l R}=-\alpha \psi_{n, l L}$. Therefore, in case of identical leads the summation over $n$ in (64) can be divided into two parts. The first part involves summation over $N+1$ solutions of Eq. (A3), while the second part involves summation over $N$ solutions of (61) such that $\Omega_{n}=E_{l}$. The first part does not depend on index $\alpha$ and it vanishes. Therefore

$$
J_{\alpha}=\mp \operatorname{Im} \sum_{l} \frac{t^{2}\left(f_{l L}-f_{l R}\right)}{\left(\varepsilon-E_{l}\right)+2 t^{2} \sum_{k} \frac{1}{\left(E_{l}-E_{k}^{*}\right)}} .
$$

Here the upper (lower) sign corresponds to $\alpha=L(\alpha=R)$. It is obvious that in the limit of macroscopically large leads $(N \rightarrow \infty, \gamma \rightarrow 0)$ eq. (A7) becomes the standard Landauer formula (67) for the current. 
1 N. Agrait, A. L. Yeyati, and J. M. van Ruitenbeek, Physics Reports 377, 81 (2003), ISSN 0370-1573.

${ }^{2}$ R. Nichols, W. Haiss, S. J. Higgins, E. Leary, S. Martin, and D. Bethell, Phys. Chem. Chem. Phys. 12, 2801 (2010).

3 Y. V. Nazarov and Y. M. Blanter, Quantum Transport: Introduction to Nanoscience (Cambridge University Press, 2009).

${ }^{4}$ M. Di Ventra, Electrical Transport in Nanoscale Systems (Cambridge University Press, 2008).

5 J. Koch and F. von Oppen, Phys. Rev. Lett. 94, 206804 (2005).

6 J. Park, A. N. Pasupathy, J. I. Goldsmith, C. Chang, Y. Jason, R. Petta, J. P. Rinkoski, M. andSethna, H. Abruna, P. L. McEuen, and D. C. Ralph, Nature 417, 722 (2002).

7 L. H. Yu and D. Natelson, Nano Letters 4, 79 (2004).

8 C. Romeike, M. R. Wegewijs, W. Hofstetter, and H. Schoeller, Phys. Rev. Lett. 96, 196601 (2006).

9 J. Chen, M. A. Reed, A. M. Rawlett, and J. M. Tour, Science 286, 1550 (1999).

${ }^{10}$ Huang, Xu, Chen, M. D. Ventra, and Tao, Nano Letters 6, 1240 (2006).

11 M. Galperin, M. A. Ratner, and A. Nitzan, Nano Letters 5, 125 (2005).

12 R. Landauer, Philos. Mag. 21, 863 (1970).

13 C. Caroli, R. Combesco, P. Nozieres, and D. Saintjam, J. Phys. C 4, 916 (1971).

14 J. Taylor, H. Guo, and J. Wang, Phys. Rev. B 6324, 245407 (2001).

15 Y. Q. Xue, S. Datta, and M. A. Ratner, Chem. Phys. 281, 151 (2002), sp. Iss. SI.

16 M. Brandbyge, J. L. Mozos, P. Ordejon, J. Taylor, and K. Stokbro, Phys. Rev. B 65, 165401 (2002).

17 M. Di Ventra, S. T. Pantelides, and N. D. Lang, Phys. Rev. Lett. 84, 979 (2000).

18 Y. Fujimoto and K. Hirose, Phys. Rev. B 67, 195315 (2003).

19 S. H. Ke, H. U. Baranger, and W. T. Yang, Phys. Rev. B 70, 085410 (2004).

20 A. Arnold, F. Weigend, and F. Evers, J. Chem. Phys. 126, 174101 (2007).

${ }^{21}$ K. Thygesen and K. Jacobsen, Chem. Phys. 319, 111 (2006).

22 Z. Li and D. S. Kosov, J. Phys.: Cond. Matt. 18, 1347 (2006).

23 A. R. Rocha, V. M. García-Suárez, S. Bailey, C. Lambert, J. Ferrer, and S. Sanvito, Phys. Rev. B 73, 085414 (2006).

24 A. Calzolari, N. Marzari, I. Souza, and M. B. Nardelli, Phys. Rev. B 69, 035108 (2004).

25 T. M. Perrine, T. Berto, and B. D. Dunietz, J. Phys. Chem. B 112, 16070 (2008).

${ }^{26}$ F. Evers, F. Weigend, and M. Koentopp, Phys. Rev. B 69, 235411 (2004).

27 Z. Li and D. S. Kosov, Phys. Rev. B 76, 035415 (2007).

28 M. Koentopp, C. Chang, K. Burke, and R. Car, J. Phys.: Cond. Matt. 20, 083203 (2008).

29 Z. Li and D. Kosov, J. Phys. Chem. B 110, 19116 (2006).

${ }^{30}$ L. Venkataraman, J. Klare, I. Tam, C. Nuckolls, M. Hybertsen, and M. Steigerwald, Nano Letters 6, 458 (2006).

31 K. S. Thygesen and A. Rubio, Phys. Rev. B 77, 115333 (2008).

32 P. Myöhänen, A. Stan, G. Stefanucci, and R. van Leeuwen, Phys. Rev. B 80, 115107 (2009).

33 C. D. Spataru, M. S. Hybertsen, S. G. Louie, and A. J. Millis, Phys. Rev. B 79, 155110 (2009).

34 Y. Dahnovsky, Phys. Rev. B 80, 165305 (2009).

35 R. Baer and D. Neuhauser, Chem. Phys. Lett. 374, 459 (2003), ISSN 0009-2614.

36 J. Cizek, Advances in Chemical Physics 14, 35 (1969).

37 R. J. Bartlett and M. Musial, Reviews of Modern Physics 79, 291 (2007).

38 A. Szabo and N. Ostlund, Modern Quantum Chemistry: Introduction to Advanced Electronic Structure Theory (Dover, 1996).

39 S. A. Gurvitz and Y. S. Prager, Phys. Rev. B 53, 15932 (1996).

40 M. Leijnse and M. R. Wegewijs, Phys. Rev. B 78, 235424 (2008).

41 U. Harbola, M. Esposito, and S. Mukamel, Phys. Rev. B 74, 235309 (2006).

42 P. Zedler, G. Schaller, G. Kiesslich, C. Emary, and T. Brandes, Phys. Rev. B 80, 045309 (2009).

43 X.-Q. Li, J. Luo, Y.-G. Yang, P. Cui, and Y. Yan, Phys. Rev. B 71, 205304 (2005).

44 J. N. Pedersen and A. Wacker, Phys. Rev. B 72, 195330 (2005).

45 I. V. Ovchinnikov and D. Neuhauser, J. Chem. Phys. 122, 024707 (pages 8) (2005).

46 J. E. Subotnik, T. Hansen, M. A. Ratner, and A. Nitzan, J. Chem. Phys. 130, 144105 (pages 12) (2009).

47 A. E. Rothman and D. A. Mazziotti, J. Chem. Phys. 132 (2010), ISSN 0021-9606.

48 G. Lindblad, Comm. Math. Phys. 48, 119 (1976).

49 D. S. Kosov, J. Chem. Phys. 131, 171102 (pages 4) (2009).

50 M. Schmutz, Z. Physik. B 30, 97 (1978).

51 U. Harbola and S. Mukamel, Physics Reports 465, 191 (2008).

52 T. Prosen, New Journal of Physics 10, 043026 (2008).

53 T. Prosen and B. Zunkovic, New Journal of Physics 12, 025016 (2010).

54 H. Matsumoto, Y. Nakano, and H. Umezawa, Phys. Rev. D 31, 429 (1985).

${ }_{55}$ H. Umezawa, Advanced Field Theory: Micro, Macro, and Thermal Physics (Springer, 1995).

${ }_{57}^{56}$ H. P. Breuer and F. Petruccione, The Theory of Open Quantum Systems (Oxford University Press, Oxford, 2002).

57 A. Kobryn, T. Hayashi, and T. Arimitsu, Annals of Physics 308, 395 (2003).

58 N. N. Bogoliubov, [Usp. Fiz. Nauk. 67, 549 (1959)] Soviet Phys. -Usp. 67, 236 (1959).

59 L. D. Landau, [Zh. Eksp. Teor. Fiz. (1956), 30, 1058] Soviet Phys. JETP 3, 920 (1957). 
60 N. N. Bogoliubov, [Zh. Eksp. Teor. Fiz. 34, 58 (1958)] Sov. Phys. JETP 7, 41 (1958).

${ }^{61}$ N. N. Bogoliubov and D. V. Shirkov, Introduction to the Theory of Quantized Fields (John Willey \& Sons, NY, 1980).

62 Y. Meir and N. S. Wingreen, Phys. Rev. Lett. 68, 2512 (1992).

63 S. Datta, Electronic Transport in Mesoscopic Systems (Cambridge University Press, 1997). 